\title{
Promoter Variant of PIK3C3 Is Associated with Autoimmunity against Ro and Sm Epitopes in African-American Lupus Patients
}

\author{
Silvia N. Kariuki, ${ }^{1}$ Beverly S. Franek, ${ }^{1}$ Rachel A. Mikolaitis, ${ }^{2}$ Tammy O. Utset, ${ }^{1}$ \\ Meenakshi Jolly, ${ }^{2}$ Andrew D. Skol, ${ }^{3}$ and Timothy B. Niewold ${ }^{1}$ \\ ${ }^{1}$ Section of Rheumatology and Gwen Knapp Center for Lupus and Immunology Research, University of Chicago, \\ 5841 S. Maryland Ave. MC 0930, Chicago, IL 60637, USA \\ ${ }^{2}$ Section of Rheumatology, Rush University Medical Center, Chicago, IL 60612, USA \\ ${ }^{3}$ Section of Genetic Medicine, University of Chicago, Chicago, IL 60637, USA \\ Correspondence should be addressed to Timothy B. Niewold, tniewold@medicine.bsd.uchicago.edu \\ Received 15 January 2010; Revised 7 May 2010; Accepted 24 May 2010 \\ Academic Editor: Brian Poole
}

Copyright ( $) 2010$ Silvia N. Kariuki et al. This is an open access article distributed under the Creative Commons Attribution License, which permits unrestricted use, distribution, and reproduction in any medium, provided the original work is properly cited.

The PIK3C3 locus was implicated in case-case genome-wide association study of systemic lupus erythematosus (SLE) which we had performed to detect genes associated with autoantibodies and serum interferon-alpha (IFN- $\alpha$ ). Herein, we examine a PIK3C3 promoter variant (rs3813065/-442 C/T) in an independent multiancestral cohort of 478 SLE cases and 522 controls. rs3813065 $\mathrm{C}$ was strongly associated with the simultaneous presence of both anti-Ro and anti-Sm antibodies in African-American patients $\left[\mathrm{OR}=2.24(1.34-3.73), P=2.0 \times 10^{-3}\right]$. This autoantibody profile was associated with higher serum IFN- $\alpha\left(P=7.6 \times 10^{-6}\right)$. In the HapMap Yoruba population, rs3813065 was associated with differential expression of ERAP2 $\left(P=2.0 \times 10^{-5}\right)$, which encodes an enzyme involved in MHC class I peptide processing. Thus, rs3813065 C is associated with a particular autoantibody profile and altered expression of an MHC peptide processing enzyme, suggesting that this variant modulates serologic autoimmunity in African-American SLE patients.

\section{Introduction}

Systemic lupus erythematosus (SLE) is a systemic autoimmune disorder characterized by involvement of multiple organ systems including the skin, musculoskeletal, renal, and hematologic systems. While the exact pathogenic mechanism of SLE is unknown, strong evidence exists for contributions from both genetic risk factors and environmental events which lead to an irreversible break in immunologic selftolerance [1]. SLE is a devastating disease, typically affecting young women during the reproductive years, resulting in significant morbidity and mortality [2]. SLE is nine times more common in women than in men [3], and four times more common in people of African-American ancestry than those of European-American ancestry [1].

Interferon-alpha (IFN- $\alpha)$ is a pleiotropic type I interferon which can bridge the innate and adaptive immune systems and thus may set thresholds for self-tolerance. Serum
IFN- $\alpha$ is elevated in many SLE patients [4]. Recombinant human IFN- $\alpha$ administered as a therapy for chronic viral hepatitis and malignancy is thought to cause SLE in some patients [5]. IFN- $\alpha$-induced SLE typically resolves after the IFN- $\alpha$ is discontinued $[6,7]$, supporting the idea that IFN$\alpha$ was causal. Serum IFN- $\alpha$ is also abnormally high in $20 \%$ of healthy first-degree relatives of SLE patients, and strong familial aggregation of high serum IFN- $\alpha$ has been observed in lupus families, supporting heritability of this trait [8]. The high IFN- $\alpha$ trait in SLE families is inherited in a complex fashion, suggesting polygenic inheritance which is not fully characterized currently [8-11].

Autoantibodies directed at double-stranded DNA (dsDNA) and RNA-binding proteins (anti-Ro, anti-La, anti-Sm, and anti-RNP, collectively anti-RBP) are characteristically found in SLE sera and are the strongest known predictors of high serum IFN- $\alpha$ in SLE patients [8]. Immune complexes formed by these autoantibodies can directly 
stimulate IFN- $\alpha$ production in vitro, likely via the endosomal Toll-like receptors [12]. These data suggest that these SLEassociated autoantibodies are correlated with and likely mechanistically related to serum IFN- $\alpha$. SLE-associated autoantibodies are also a heritable trait in SLE families [13], and a number of established SLE-risk loci have demonstrated associations with autoantibodies [14-16].

We have recently conducted a case-case genome-wide association study (GWAS) to determine the genetic factors associated with SLE- associated autoantibodies and high serum IFN- $\alpha$ (Kariuki et al., submitted). In this GWAS study, four SNPs which were all in very high LD $(r$-squared $>0.8)$ in the $5^{\prime}$ region near the PIK3C3 gene were in the top 200 SNPs which showed evidence for association with autoantibody traits and/or serum IFN- $\alpha$. Phosphoinositide-3-kinase, class 3 (PIK3C3), is a member of the phosphatidylinositide 3 -kinase family that maps to chromosome 18q12. The PIK3C3 protein, also known as hVps34, plays a role in vacuolar sorting, and a homolog of this protein in yeast exhibits similar functions [17]. Recent studies have shown that PIK3C3 associates with Beclin-1 and is an important regulator of autophagy [18].

We searched the literature for any previous genetic associations between PIK3C 3 and human disease and discovered that a functional variant in the promoter region (rs3813065/$442 \mathrm{C} / \mathrm{T}$ ) was associated with susceptibility to schizophrenia and bipolar disease $[19,20]$. Neuropsychiatric manifestations of SLE are common, ranging from mild cognitive deficits to seizures and psychosis. These data suggest the possibility of pathogenic overlap between schizophrenia and SLE. Also, a previous study demonstrated increased rates of anti-Sm autoantibodies in both schizophrenic patients and their healthy relatives, suggesting both heritability and association of this autoantibody with schizophrenia [21]. This finding is of particular interest, as the anti-Sm immune response is thought to be highly specific for SLE, and like other SLE-associated autoantibodies anti-Sm is a heritable trait in SLE families [13]. These data taken together suggest that some shared heritable elements could contribute to this particular serologic autoimmunity found in both SLE and schizophrenia. It was hence interesting to find the PIK3C3 locus which has previously been linked to schizophrenia susceptibility represented in our list of top 200 SNPs in our GWAS which were associated with SLE serology and IFN- $\alpha$ levels. The SNPs in PIK3C3 which were found in our GWAS study were located in the $5^{\prime}$ region of the gene characterized by high LD, and we chose to study the functional promoter variant of PIK3C3 in an independent cohort of lupus patients and controls in the present study to test for association with SLE susceptibility, as well as potential associations with serology and serum IFN- $\alpha$ levels.

\section{Methods}

2.1. Initial GWAS Study Description. The initial cohort of SLE patients studied in the GWAS scan was obtained from the Hospital for Special Surgery Lupus Registries, and consisted of 104 SLE patients, including 20 African-American, 36
European-American, 32 Hispanic-American, and 16 AsianAmerican SLE patients. This study was designed as a casecase analysis to compare SNP frequencies in SLE patients with high versus low IFN- $\alpha$ and those with and without SLE-associated autoantibodies. Patients were selected in an extremes-of-phenotype design from the top 33\% and bottom $33 \%$ of serum IFN- $\alpha$ activity and were additionally stratified for the GWAS study by ancestry and the presence or absence of anti-RBP or anti-dsDNA antibodies. A study design incorporating multiple ancestral backgrounds was chosen as both autoantibodies and serum IFN- $\alpha$ levels are heritable pathogenic factors which are shared between all ancestral backgrounds. The top 200 SNPs were examined in detail using expert review of public databases, and the seven top SNPs chosen for replication all demonstrated strong associations with either serology or serum IFN- $\alpha$ in an independent cohort, as would have been expected based upon the initial GWAS study design (Kariuki et al., in revision). An SNP $5^{\prime}$ to the PIK3C3 gene was also noted as an SNP of interest among the top 200 SNPs in this GWAS study. Based upon the data presented in the introduction, the functional promoter variant $5^{\prime}$ of PIK3C 3 was chosen for follow up in this study.

2.2. Validation Cohort. The independent validation cohort of 478 SLE patients was obtained from the University of Chicago Translational Research in the Department of Medicine (TRIDOM) registry and Rush University Medical Center and consisted of 252 African-American, 152 European-American, and 74 Hispanic-American SLE patients. All patients met the revised 1982 ACR criteria for the diagnosis of SLE [22]. Samples from 522 controls were obtained from the TRIDOM registry, including 361 AfricanAmerican and 161 European-American subjects who were individually screened for the absence of autoimmune disease by medical record review. The subjects in this study were not related to each other. Informed consent was obtained from all subjects at each site, and the study was approved by the IRB at each institution.

\subsection{SNP Genotyping of PIK3C3 rs3813065 in the Validation} Cohort. Individuals in the validation cohort were genotyped at PIK3C3 rs3813065. Genotyping was performed using ABI Taqman Assays-by-Design primers and probes on an ABI 7900HT PCR machine with $>98 \%$ genotyping success. Scatter plots were all reviewed individually for quality, and genotype frequencies did not deviate significantly from the expected Hardy-Weinberg proportions $(P>.01$ in all ancestral backgrounds).

2.4. Reporter Cell Assay for IFN- $\alpha$. The reporter cell assay for IFN- $\alpha$ has been described in detail elsewhere $[8,23]$. Reporter cells were used to measure the ability of patient sera to cause IFN-induced gene expression. The reporter cells (WISH cells, ATCC no. CCL-25) were cultured with 50\% patient sera for 6 hours, and then lysed. mRNA was purified from cell lysates, and cDNA was made from total cellular mRNA. cDNA was then quantified using real-time PCR 
TABLE 1: Percent of SLE patients with a positive test for each SLEassociated autoantibody in each ancestral background.

\begin{tabular}{lcccccc}
\hline & Ro & La & Sm & RNP & DNA & Ro and Sm \\
\hline Af-Am & 52 & 19 & 38 & 65 & 41 & 24 \\
His & 40 & 10 & 22 & 33 & 48 & 14 \\
Eur-Am & 33 & 9 & 13 & 20 & 43 & 4 \\
\hline
\end{tabular}

Af-Am: African-American, His: Hispanic-American, and Eur-Am: European-American, Numbers indicate percent of subjects with a positive test in each category; "Ro and Sm" category indicates subjects with positive tests for both autoantibodies.

using an Applied Biosystems 7900HT PCR machine with the SYBR Green fluorophore system. Forward and reverse primers for the genes MX1,PKR, and IFIT1, which are known to be highly and specifically induced by IFN- $\alpha$, were used in the reaction [8]. GAPDH was amplified in the same samples to control for background gene expression.

The amount of PCR product of the IFN- $\alpha$-induced gene was normalized to the amount of product for the housekeeping gene GAPDH in the same sample. The relative expression of each of the three tested IFN-induced genes was calculated as a fold increase compared to its expression in WISH cells cultured with media alone. Results from the IFN- $\alpha$ assay were standardized to a healthy multiancestral reference population as previously described, and a serum IFN- $\alpha$ activity score was calculated based upon the mean and SD of the reference population [8]. This assay has been highly informative when applied to SLE as well as other autoimmune disease populations $[8,24,25]$.

2.5. Measurement of Autoantibodies. Antibodies to anti-Ro, anti-La, anti-Sm, and anti-RNP were measured in all samples by ELISA methods using kits from INOVA Diagnostics (San Diego, CA), and anti-dsDNA antibodies were measured using Crithidia luciliae immunofluorescence, with detectable fluorescence considered positive. All samples were assayed in University of Chicago clinical laboratory by the same personnel that test clinical samples. For the ELISA assays, the standard cutoff points for a positive test designated by the manufacturer were used to categorize samples as positive or negative. Autoantibody prevalences in SLE patients of each ancestral background are shown in Table 1.

2.6. Statistical Analysis. All subjects in the study had genotype data available for 13 SNPs, and principal component analysis was performed on 12 of these 13 SNPs excluding the PIK3C3 rs3813065 SNP, using the PCA option in the Cluster program by Eisen et al. [26]. The first two principal components are shown plotted on the $X$ and $Y$ axes, respectively, in Figure 1, and the first component provides a strong separation of those subjects of selfreported African-American ancestry from those of selfreported European-American ancestry. If the zero point on the $X$-axis of Figure 1 was used to categorically split the population, $84.4 \%$ of self-reported European ancestry

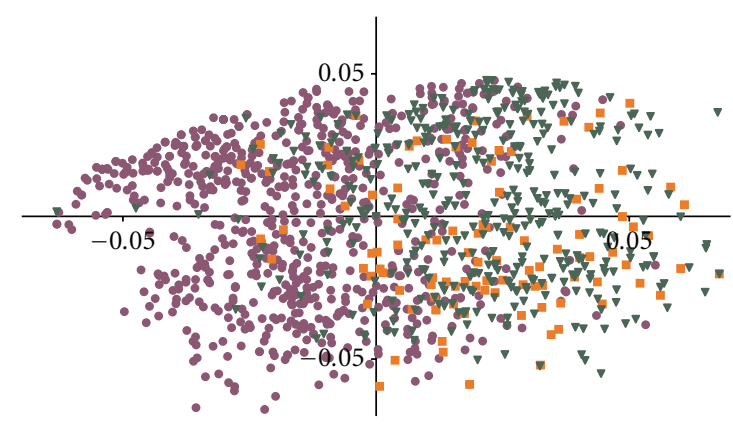

- AA

- His

- EA

Figure 1: Principal component (PC) analysis including all cases and controls. A. shows the first PC ( $x$-axis) versus the second PC ( $y$-axis) for each subject. Each dot represents one subject and is color-coded by self-reported ancestral background (AA: AfricanAmerican, His : Hispanic American, EA : European-American).

subjects fell in the positive range for the first principal component, and $74.3 \%$ of self-reported African-American subjects had a negative value for the first principal component $(P$-value for departure from a random distribution of these two ancestral populations on the $X$-axis $=2.8 \times 10^{-83}$ ). We included the first principal component as a covariate in all subsequent association analyses to provide control for differences in proportional ancestry in both cases and controls.

Logistic regression models were used to detect associations between the presence of autoantibodies and genotype at rs3813065 using the PLINK program v1.07 [27] (http://pngu.mgh.harvard.edu/ purcell/plink/). All logistic regressions incorporated the first principal component as a covariate to provide some control for genetic ancestry at the individual level. Testing for homogeneity of odds ratios obtained in the study was performed using PLINK with the partitioning of the chi-square test statistic method. The IFN$\alpha$ data was nonnormally distributed, and nonparametric Mann-Whitney $U$ was used to compare quantitative IFN$\alpha$ data between two genotype subgroups. When more than two columns of quantitative data were compared, the nonparametric one-way ANOVA (Kruskall-Wallis test) was used to test the hypothesis that the distribution in at least one column was significantly different from the distribution in the other columns. Chi-square test was used to compare allele frequency data between cases and controls. $P$-values shown in the paper are uncorrected for multiple comparisons. When testing differences in allele frequencies between different patient groups defined by autoantibodies, a $P$-value of .0071 is sufficient to control the family-wise type I error rate at 0.05 . For the serum IFN- $\alpha$ studies in African-American SLE patients, $P$ values $<.0056$ would withstand a Bonferroni correction for the number of comparisons possible between the different autoantibody states and genotypes presented on the graph. 


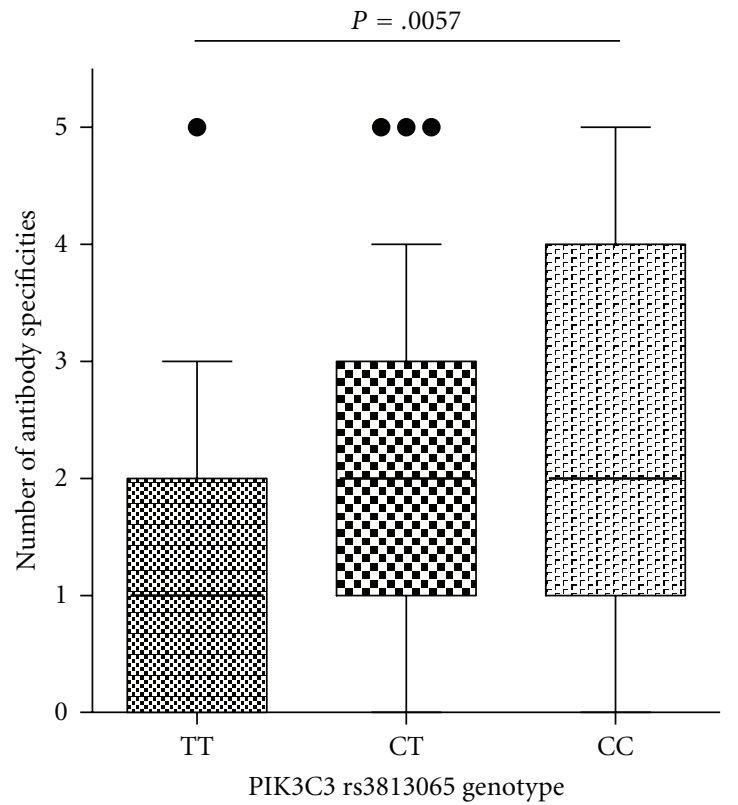

Figure 2: Distribution of the number of different autoantibody specificities in African-American SLE patients stratified by rs3813065 genotype. no. of specificities = the sum of the number of positive test results for anti-Ro, anti-La, anti-Sm, anti-RNP, and anti-dsDNA for a given subject (thus every subject is assigned a number 0 through 5 ). The horizontal line in the middle of the box indicates the median, boundaries of the boxes indicate the interquartile range, and the error bars show the 10th and 90th percentile, respectively. The $P$ value for the Kruskall-Wallis test is shown.

2.7. SCAN Database Query. We searched rs3813065 in the SNP and CNV Annotation (SCAN) database (http://www.scandb.org/) [28]. This database is a searchable index of genome-wide gene expression data linked to genome-wide SNP genotype data from the HapMap project. Gene expression data is derived from studies in which gene expression arrays were run on Epstein-Barr virustransformed lymphoblastoid cell lines from individuals genotyped in the HapMap project. The SCAN database contains expression data from both European (Centre d'Etude du Polymorphisme Humain or CEPH) and West African (Yoruba or YRI) HapMap reference populations. We searched the database using rs3813065 as the query term, with a threshold $P$ value of $10^{-4}$.

\section{Results}

3.1. Association of rs3813065 with Autoantibody Specificities. We detected an association between the $\mathrm{C}$ allele of rs3813065 and increasing number of autoantibody specificities in African-American SLE patients $(P=.0057$, Figure 2). The number of antibody specificities was calculated as the sum of the number of positive results for anti-Ro, anti-La, anti-Sm, anti-RNP, and anti-dsDNA antibody testing in a given SLE patient, for a maximum of 5 distinct antibody specificities. This $P$ value of .0057 would exceed a Bonferroni correction for the number of specificities analyzed. There were similar nonsignificant trends in the Hispanic-American and European-American cohorts $(P=.23$ and.32, resp., data not shown).

3.2. Association of rs3813065 with Individual Autoantibodies. Logistic regression models were run to detect associations between rs3813065 C and each SLE-associated autoantibody individually and follow up models assessed associations with autoantibody combinations. Both anti-Ro and antiSm showed evidence for association with rs3813065 C allele in African-Americans, and the best fit model was achieved between the rs3813065 C and the combination of anti-Ro and anti-Sm antibodies in this background (Table $1, \mathrm{OR}=2.24,95 \% \mathrm{CI}=1.34-3.73$, and $P=2.0 \times 10^{-3}$ ). This association clearly withstands Bonferroni correction for the number of comparisons in this study. A similar nonsignificant trend was observed for an association between rs3813065 C and the combination of anti-Ro and anti-Sm antibodies in Hispanic-American and European-American subjects $(\mathrm{OR}=3.30, P=.14$ and $\mathrm{OR}=2.22, P=.45$, resp., Table 2). While the direction and magnitude of the odds ratio for association was similar in each background, independent statistical significance was not observed in the Hispanic- and European-American subjects due to the low prevalence of the anti-Ro/anti-Sm serologic profile (Table 1). The odds ratios for association of the rs 3813065 $\mathrm{C}$ allele with the anti-Ro/anti-Sm antibody profile in each individual ancestral background were not significantly different $\left(\chi^{2}=0.21, P=.90\right)$. A meta-analysis across all three ancestral backgrounds supported a significant overall association $(\mathrm{OR}=2.26,95 \% \mathrm{CI}=1.43-3.55$, and $P=5.0 \times$ $10^{-4}$ ) although most of data supporting this meta-analysis association is derived from the African-American subjects in whom this antibody profile is the most prevalent.

3.3. Association of rs3813065 with Serum IFN- $\alpha$ Activity in African-Americans. Genetic variation at the rs3813065 SNP was not associated independently with differences in serum IFN- $\alpha$, however the African-American SLE patients who had both anti-Ro and anti-Sm had higher serum IFN- $\alpha$ than those SLE patients who lacked this antibody profile (Figure 3, $\left.P=7.6 \times 10^{-6}\right)$. These data support a secondary association with serum IFN- $\alpha$, in which genetic variation is associated with the anti-Ro/anti-Sm antibody profile, and then the particular serologic profile is associated with increased serum IFN- $\alpha$.

3.4. Case-Control Association Study of PIK3C3 rs3813065. There were no significant differences in allele frequencies between cases and controls in our cohort although the $\mathrm{C}$ allele trended toward enrichment in the African-American cases as compared to controls (Table 3, $\mathrm{OR}=1.07, P=.63$ ). rs3813065 C allele frequency in African-American cases lacking either anti-Ro or anti-Sm antibodies was similar to African-American controls (0.605 versus 0.620, resp.), supporting the idea that the $\mathrm{C}$ allele is specifically enriched with the subgroup of patients who have both anti-Ro and anti-Sm antibodies. 
TABLE 2: Association of the rs3813065 C allele with anti-Ro and anti-Sm positive patients in each ancestral background.

\begin{tabular}{|c|c|c|c|c|c|}
\hline & Antibody profile & MAF & OR (C allele) & $95 \mathrm{CI}$ & $P$ value \\
\hline \multirow{2}{*}{ African-american } & $\mathrm{Ro}+\mathrm{Sm}+\mathrm{SLE}$ & 0.245 & \multirow{2}{*}{2.24} & \multirow{2}{*}{$1.34-3.73$} & \multirow{2}{*}{$2.0 \times 10^{-3}$} \\
\hline & All other SLE & 0.395 & & & \\
\hline \multirow{2}{*}{ Hispanic-american } & $\mathrm{Ro}+\mathrm{Sm}+\mathrm{SLE}$ & 0.119 & \multirow{2}{*}{3.30} & \multirow{2}{*}{$0.67-16.25$} & \multirow{2}{*}{.14} \\
\hline & All other SLE & 0.248 & & & \\
\hline \multirow{2}{*}{ European-american } & $\mathrm{Ro}+\mathrm{Sm}+\mathrm{SLE}$ & 0.115 & \multirow{2}{*}{2.21} & \multirow{2}{*}{$0.28-17.67$} & \multirow{2}{*}{.45} \\
\hline & All other SLE & 0.160 & & & \\
\hline Meta-analysis & $\begin{array}{l}\text { Ro+ Sm+ SLE versus } \\
\text { all other SLE }\end{array}$ & - & 2.26 & $1.43-3.55$ & $5.0 \times 10^{-4}$ \\
\hline
\end{tabular}

MAF: minor allele frequency, OR: odds ratio for association of the $\mathrm{C}$ allele with Ro+Sm+ patients as compared to all other SLE patients, and $95 \mathrm{CI}=95 \%$ confidence interval of the odds ratio.

TABLE 3: Case-control association of rs3813065 with SLE in Africanand European-American subjects.

\begin{tabular}{lcccc}
\hline & MAF & OR (C allele) & 95 CI & $P$ value \\
\hline AA cases & 0.320 & 1.07 & $0.82-1.38$ & .63 \\
AA controls & 0.380 & & & \\
\hline EA cases & 0.155 & 0.65 & $0.40-1.06$ & .09 \\
EA controls & 0.115 & & & \\
\hline
\end{tabular}

MAF: minor allele frequency, OR: odds ratio for association of the $\mathrm{C}$ allele in SLE cases as compared to controls in each ancestral background, and 95 $\mathrm{CI}=95 \%$ confidence interval of the odds ratio.

3.5. Association of PIK3C3 rs3813065 with ERAP2 Expression in the SCAN Database. We next queried the SCAN database (http://www.scandb.org/) to determine whether genetic variation at the rs3813065 SNP was associated with any significant differences in global gene expression in EBVtransformed lymphoblastoid cell lines derived from HapMap individuals [28]. Interestingly, the rs3813065 SNP was associated with a highly significant difference in the expression of endoplasmic reticulum aminopeptidase 2 (ERAP2) in the HapMap cell lines derived from the African Yoruba population $\left(P=2 \times 10^{-5}\right)$. The ERAP2 gene is located on chromosome $5 \mathrm{q} 15$ and the molecular mechanism underlying this association is not known. ERAP2 is expressed in the endoplasmic reticulum, and is involved in precursor peptide processing prior to binding to HLA class I molecules in the endoplasmic reticulum [29]. No other transcript levels were associated with rs3813065, including PIK3C3 itself, in the Yoruba population. There were no associations between variation at rs3813065 and gene expression levels in lymphoblastoid cells from the representative Europeanderived CEPH HapMap population.

\section{Discussion}

We find that genetic variation in the promoter region of the PIK3C3 gene is associated with a very particular serologic phenotype in African-American SLE patients. It is interesting that we find a primary association with serology and not with serum IFN- $\alpha$ levels, although autoantibodies may be more highly heritable than IFN- $\alpha$. In SLE families, anti-Ro

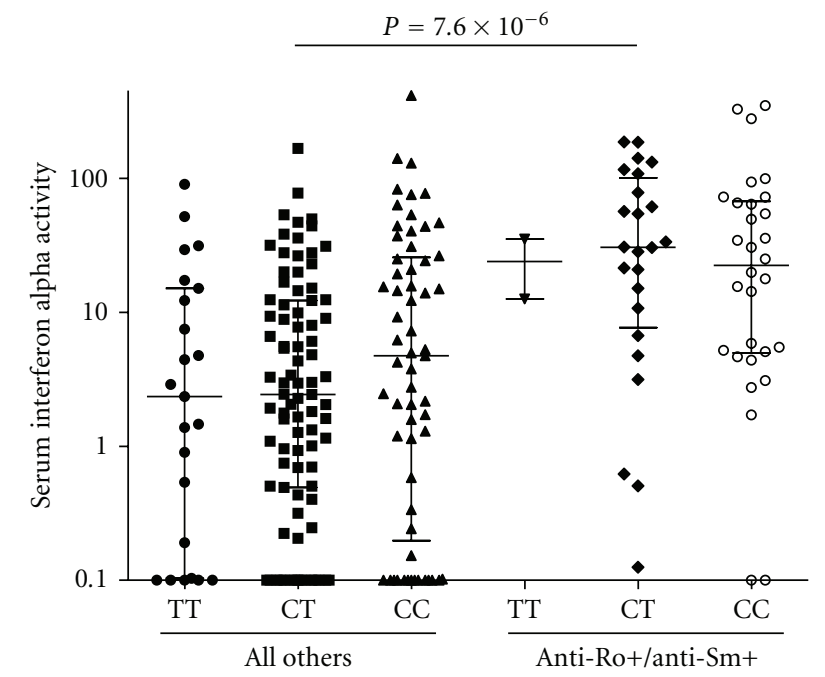

Figure 3: Serum IFN- $\alpha$ activity in African-American SLE patients stratified by rs3813065 genotype and presence or absence of the anti-Ro positive/anti-Sm positive serologic phenotype. Serum IFN$\alpha$ is represented in relative units on the $Y$-axis, as described in Methods. "Anti-Ro+/Anti-Sm+" indicates SLE patients who have positive tests for both anti-Ro and anti-Sm, and "All others" indicates all of the other SLE patients who lack one or both of these autoantibodies. Line drawn at the median and error bars show the interquartile range. $P$ value by Mann-Whitney $U$ test for a difference between subjects with CT genotype with versus without the anti-Ro+/anti-Sm+ serology.

antibodies are characterized by a relative recurrence risk of 9.7 [13] while high IFN- $\alpha$ has a relative recurrence risk of 3.8 [8]. Relative recurrence risk for anti-Sm in SLE families is incalculable in the same large study [13], as none of the 877 controls had a positive result for anti-Sm. However, $0.2 \%$ of the 819 unaffected relatives of SLE patients had a positive anti-Sm test, supporting probable familial aggregation of this autoantibody trait as well.

The rs3813065 SNP has been associated with schizophrenia in multiple ancestral backgrounds $[19,20]$. In the largest study to date in Chinese individuals, the rs3813065 $\mathrm{C}$ allele was associated with increased risk of schizophrenia $(\mathrm{OR}=1.24, P=.017)[20]$, and a Chinese family-based 
association study demonstrated similar findings $(\mathrm{OR}=1.51$, $P=.0036$ for association of the $\mathrm{C}$ allele) [30]. A study examining the rs3813065 allele in European ancestry populations did not find evidence for an association with schizophrenia while there was some evidence for association between the opposite allele ( $\mathrm{T}$ allele) with risk of schizophrenia in a Jewish population [19]. Thus, while published data support an association between this locus and schizophrenia, there appears to be significant heterogeneity of association between different world populations. SNPs in or near the PIK3C3 locus have not been associated with SLE in genomewide studies to date; however these studies have only assessed European ancestry individuals and have not stratified by subphenotypes such as autoantibody traits.

Studies have implicated polygenic and environmental causes for both SLE [31] and schizophrenia [32, 33]. Infection with the Epstein-Barr virus (EBV) has been strongly implicated as an environmental risk factor for SLE [34], and the herpes virus family may play a role in the development of schizophrenia $[35,36]$. Studies suggest that formation of the characteristic SLE-associated autoantibodies anti-Sm and anti-Ro can be triggered by antigenic cross-reactivity with anti-Epstein-Barr virus nuclear antigen-1 (EBNA-1) antibodies [31, 37]. Work in mice and rabbits demonstrated that immunization with EBNA-1 peptides can elicit the production of anti-Sm and anti-RNP autoantibodies [38]. Interestingly, in our study the $C$ allele of the rs3813065 SNP was strongly associated with anti-Ro combined with anti-Sm antibodies in African-American SLE patients. Studies have shown that anti-Sm antibodies are also elevated in patients with schizophrenia and their healthy family members [21]; however it is not known whether this polymorphism is associated with anti-Sm antibodies in schizophrenia patients.

The strong association between the rs3813065 SNP and ERAP2 expression is of particular interest. ERAP2 is expressed in the endoplasmic reticulum and is involved in the processing of precursor peptides prior to their binding to HLA class I molecules in the endoplasmic reticulum [29]. HLA class I molecules present cytosolic peptides, including peptides derived from viruses, allowing presentation of antigens to the adaptive immune system and subsequent development of an immune response directed against the particular peptide. It is possible that dysregulation in ERAP2 expression due to variation in the PIK3C3 gene could lead to alternate peptide processing and presentation on MHC molecules. Improper peptide processing could enhance the chances of anti-self-immune response. Thus, the association of rs3813065 C with the formation of particular autoantibodies in SLE patients could be related to alternate ERAP2-mediated peptide processing prior to presentation on MHC molecules. This could begin to explain the curious finding of anti-Sm antibodies in schizophrenia patients and their family members although the data presented in this paper are only statistically significant in AfricanAmericans, and the PIK3C3 SNP has not been definitively associated with schizophrenia in African-Americans [39]. Studies examining whether this polymorphism is related to anti-Sm serology in schizophrenia patients, and studies to determine whether there is any association between this variant and neuropsychiatric manifestations of SLE will be important areas for future work.

\section{Acknowledgment}

The authors declared that there is no conflict of interests. T. O. Utset, Lupus Clinical Trials Consortium, received consulting fees from Genentech; T. B. Niewold, from NIH K08 AI083790, NIAID Clinical Research Loan Repayment AI071651, NIH CTSA Core Subsidy Grant, and CTSA Pilot Grants from UL1 RR024999, Lupus Research Institute Novel Research Grant, Alliance for Lupus Research Target Identification in Lupus Grant, Arthritis National Research Foundation Eng Tan Scholar Award.

\section{References}

[1] J. B. Harley, J. A. Kelly, and K. M. Kaufman, "Unraveling the genetics of systemic lupus erythematosus," Springer Seminars in Immunopathology, vol. 28, no. 2, pp. 119-130, 2006.

[2] A. Ippolito and M. Petri, "An update on mortality in systemic lupus erythematosus," Clinical and Experimental Rheumatol$o g y$, vol. 26, no. 5, supplement 51, pp. S72-S79, 2008.

[3] C. E. Weckerle and T. B. Niewold, "The unexplained female predominance of systemic lupus erythematosus: clues from genetic and cytokine studies," Clinical Reviews in Allergy and Immunology. Online first.

[4] J. J. Hooks, H. M. Moutsopoulos, and S. A. Geis, "Immune interferon in the circulation of patients with autoimmune disease," The New England Journal of Medicine, vol. 301, no. 1, pp. 5-8, 1979.

[5] L. E. Ronnblom, K. E. Oberg, and G. V. Alm, "Possible induction of systemic lupus erythematosus by interferon $\alpha$ treatment in a patient with a malignant carcinoid tumour," Journal of Internal Medicine, vol. 227, no. 3, pp. 207-210, 1990.

[6] T. B. Niewold and W. I. Swedler, "Systemic lupus erythematosus arising during interferon-alpha therapy for cryoglobulinemic vasculitis associated with hepatitis C," Clinical Rheumatology, vol. 24, no. 2, pp. 178-181, 2005.

[7] T. B. Niewold, "Interferon alpha-induced lupus: proof of principle," Journal of Clinical Rheumatology, vol. 14, no. 3, pp. 131-132, 2008.

[8] T. B. Niewold, J. Hua, T. J. A. Lehman, J. B. Harley, and M. K. Crow, "High serum IFN- $\alpha$ activity is a heritable risk factor for systemic lupus erythematosus," Genes and Immunity, vol. 8, no. 6, pp. 492-502, 2007.

[9] S. N. Kariuki, M. K. Crow, and T. B. Niewold, "The PTPN22 C1858T polymorphism is associated with skewing of cytokine profiles toward high interferon- $\alpha$ activity and low tumor necrosis factor $\alpha$ levels in patients with lupus," Arthritis and Rheumatism, vol. 58, no. 9, pp. 2818-2823, 2008.

[10] S. N. Kariuki, K. A. Kirou, E. J. MacDermott, L. Barillas-Arias, M. K. Crow, and T. B. Niewold, "Cutting edge: autoimmune disease risk variant of STAT4 confers increased sensitivity to IFN-alpha in lupus patients in vivo," Journal of Immunology, vol. 182, no. 1, pp. 34-38, 2009.

[11] S. N. Kariuki and T. B. Niewold, "Genetic regulation of serum cytokines in systemic lupus erythematosus," Translational Research, vol. 155, no. 3, pp. 109-117, 2010.

[12] T. Lövgren, M.-L. Eloranta, B. Kastner, M. Wahren-Herlenius, G. V. Alm, and L. Rönnblom, "Induction of interferon- $\alpha$ by immune complexes or liposomes containing systemic lupus erythematosus autoantigen- and Sjögren's syndrome auto 
antigen-associated RNA," Arthritis and Rheumatism, vol. 54, no. 6, pp. 1917-1927, 2006.

[13] P. S. Ramos, J. A. Kelly, C. Gray-McGuire et al., "Familial aggregation and linkage analysis of autoantibody traits in pedigrees multiplex for systemic lupus erythematosus," Genes and Immunity, vol. 7, no. 5, pp. 417-432, 2006.

[14] K. E. Taylor, E. F. Remmers, A. T. Lee et al., "Specificity of the STAT4 genetic association for severe disease manifestations of systemic lupus erythematosus," PLoS Genetics, vol. 4, no. 5, Article ID e1000084, 2008.

[15] S. N. Kariuki, J. G. Moore, K. A. Kirou, M. K. Crow, T. O. Utset, and T. B. Niewold, "Age- and gender-specific modulation of serum osteopontin and interferon- $\alpha$ by osteopontin genotype in systemic lupus erythematosus," Genes and Immunity, vol. 10, no. 5, pp. 487-494, 2009.

[16] R. Salloum, B. S. Franek, S. N. Kariuki et al., "Genetic variation at the IRF7/PHRF1 locus is associated with autoantibody profile and serum interferon- $\alpha$ activity in lupus patients," Arthritis and Rheumatism, vol. 62, no. 2, pp. 553-561, 2010.

[17] J. M. Backer, "The regulation and function of Class III PI3Ks: novel roles for Vps34," Biochemical Journal, vol. 410, no. 1, pp. $1-17,2008$.

[18] A. Simonsen and S. A. Tooze, "Coordination of membrane events during autophagy by multiple class III PI3-kinase complexes," The Journal of Cell Biology, vol. 186, no. 6, pp. 773-782, 2009.

[19] P. Stopkova, T. Saito, D. F. Papolos et al., "Identification of PIK3C3 promoter variant associated with bipolar disorder and schizophrenia," Biological Psychiatry, vol. 55, no. 10, pp. 981988, 2004.

[20] R. Tang, X. Zhao, C. Fang et al., "Investigation of variants in the promoter region of PIK3C3 in schizophrenia," Neuroscience Letters, vol. 437, no. 1, pp. 42-44, 2008.

[21] P. Sirota, M. Firer, K. Schild et al., "Increased anti-Sm antibodies in schizophrenic patients and their families," Progress in Neuro-Psychopharmacology and Biological Psychiatry, vol. 17, no. 5, pp. 793-800, 1993.

[22] E. M. Tan, A. S. Cohen, J. F. Fries, et al., "The 1982 revised criteria for the classification of systemic lupus erythrematosus," Arthritis and Rheumatism, vol. 25, no. 11, pp. 1271-1277, 1982.

[23] J. Hua, K. Kirou, C. Lee, and M. K. Crow, "Functional assay of type I interferon in systemic lupus erythematosus plasma and association with anti-RNA binding protein autoantibodies," Arthritis and Rheumatism, vol. 54, no. 6, pp. 1906-1916, 2006.

[24] T. B. Niewold, T. L. Rivera, J. P. Buyon, and M. K. Crow, "Serum type I interferon activity is dependent on maternal diagnosis in anti-SSA/Ro-positive mothers of children with neonatal lupus," Arthritis and Rheumatism, vol. 58, no. 2, pp. 541-546, 2008.

[25] T. B. Niewold, S. N. Kariuki, G. A. Morgan, S. Shrestha, and L. M. Pachman, "Elevated serum interferon- $\alpha$ activity in juvenile dermatomyositis: associations with disease activity at diagnosis and after thirty-six months of therapy," Arthritis and Rheumatism, vol. 60, no. 6, pp. 1815-1824, 2009.

[26] M. B. Eisen, P. T. Spellman, P. O. Brown, and D. Botstein, "Cluster analysis and display of genome-wide expression patterns," Proceedings of the National Academy of Sciences of the United States of America, vol. 95, no. 25, pp. 14863-14868, 1998.

[27] S. Purcell, B. Neale, K. Todd-Brown et al., "PLINK: a tool set for whole-genome association and population-based linkage analyses," American Journal of Human Genetics, vol. 81, no. 3, pp. 559-575, 2007.
[28] E. R. Gamazon, W. Zhang, A. Konkashbaev et al., "SCAN: SNP and copy number annotation," Bioinformatics, vol. 26, no. 2, pp. 259-262, 2009.

[29] D. Fruci, P. Giacomini, M. R. Nicotra et al., "Altered expression of endoplasmic reticulum aminopeptidases ERAP1 and ERAP2 in transformed non-lymphoid human tissues," Journal of Cellular Physiology, vol. 216, no. 3, pp. 742-749, 2008.

[30] S. Duan, R. Gao, Q. Xing et al., "A family-based association study of schizophrenia with polymorphisms at three candidate genes," Neuroscience Letters, vol. 379, no. 1, pp. 32-36, 2005.

[31] J. B. Harley and J. A. James, "Epstein-Barr virus infection induces lupus autoimmunity," Bulletin of the NYU Hospital for Joint Diseases, vol. 64, no. 1-2, pp. 45-50, 2006.

[32] I. I. Gottesman and J. Shields, "A polygenic theory of schizophrenia," Proceedings of the National Academy of Sciences of the United States of America, vol. 58, no. 1, pp. 199-205, 1967.

[33] S. M. Purcell, N. R. Wray, J. L. Stone et al., "Common polygenic variation contributes to risk of schizophrenia and bipolar disorder," Nature, vol. 460, no. 7256, pp. 748-752, 2009.

[34] J. A. James, K. M. Kaufman, A. D. Farris, E. Taylor-Albert, T. J. A. Lehman, and J. B. Harley, "An increased prevalence of Epstein-Barr virus infection in young patients suggests a possible etiology for systemic lupus erythematosus," Journal of Clinical Investigation, vol. 100, no. 12, pp. 3019-3026, 1997.

[35] L. E. Delisi, S. B. Smith, and J. R. Hamovit, "Herpes simplex virus, cytomegalovirus and Epstein-Barr virus antibody titres in sera from schizophrenic patients," Psychological Medicine, vol. 16, no. 4, pp. 757-763, 1986.

[36] C. J. Carter, "Schizophrenia susceptibility genes directly implicated in the life cycles of pathogens: cytomegalovirus, influenza, herpes simplex, rubella, and Toxoplasma gondii," Schizophrenia Bulletin, vol. 35, no. 6, pp. 1163-1182, 2009.

[37] B. D. Poole, R. H. Scofield, J. B. Harley, and J. A. James, "Epstein-Barr virus and molecular mimicry in systemic lupus erythematosus," Autoimmunity, vol. 39, no. 1, pp. 63-70, 2006.

[38] B. D. Poole, T. Gross, S. Maier, J. B. Harley, and J. A. James, "Lupus-like autoantibody development in rabbits and mice after immunization with EBNA-1 fragments," Journal of Autoimmunity, vol. 31, no. 4, pp. 362-371, 2008.

[39] T. Saito, M. R. Aghalar, and H. M. Lachman, "Analysis of PIK3C3 promoter variant in African-Americans with schizophrenia," Schizophrenia Research, vol. 76, no. 2-3, pp. 361-362, 2005. 


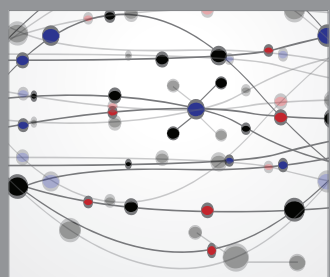

The Scientific World Journal
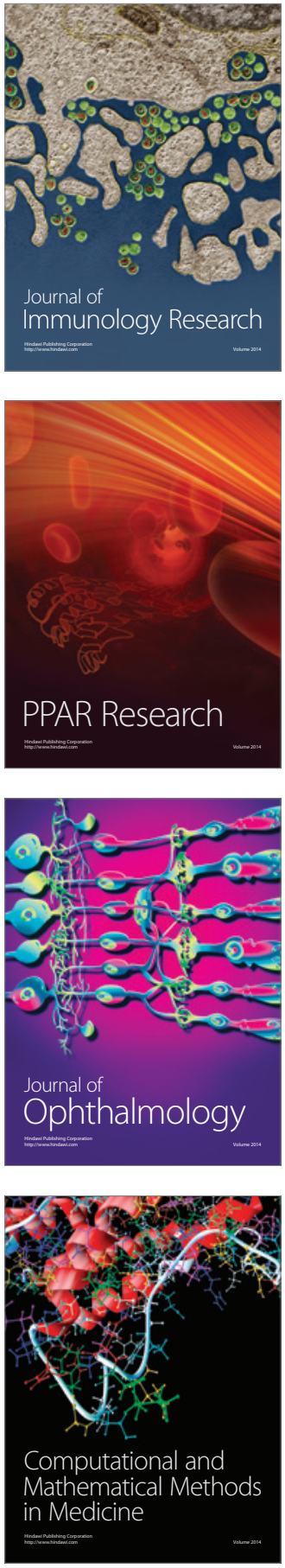

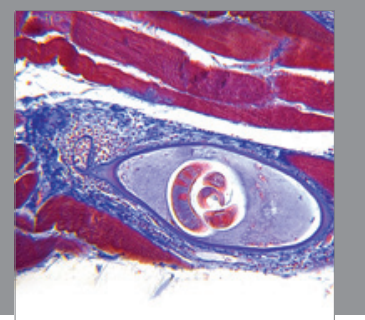

Gastroenterology

Research and Practice
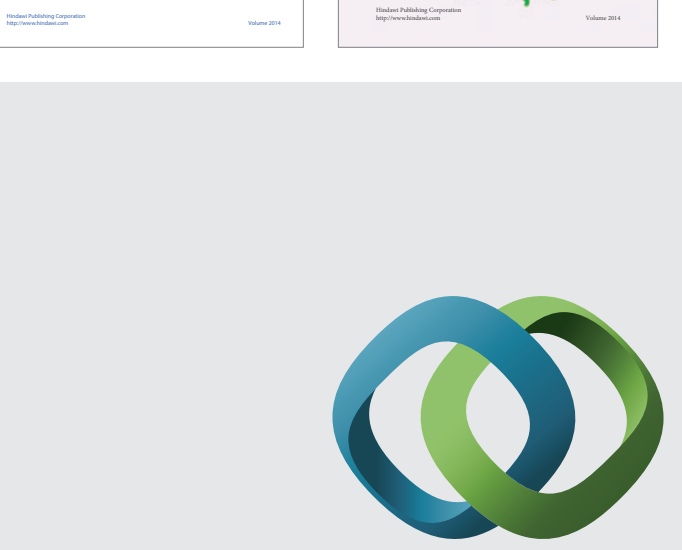

\section{Hindawi}

Submit your manuscripts at

http://www.hindawi.com
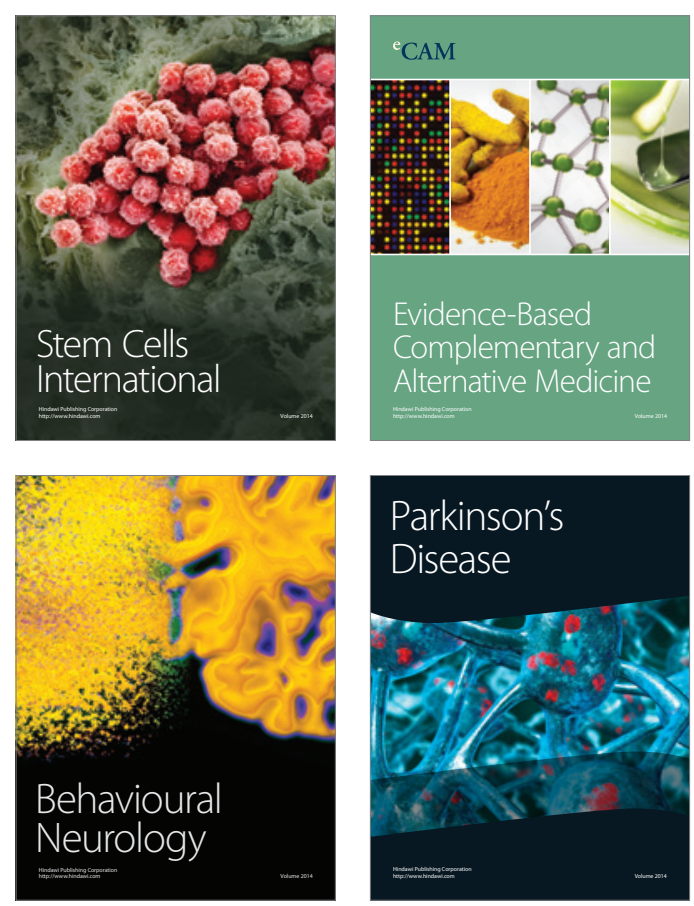

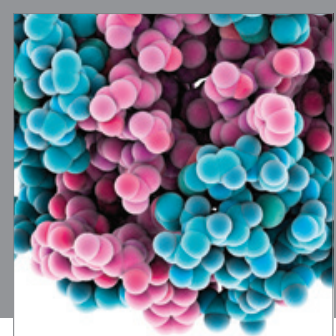

Journal of
Diabetes Research

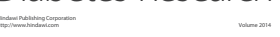

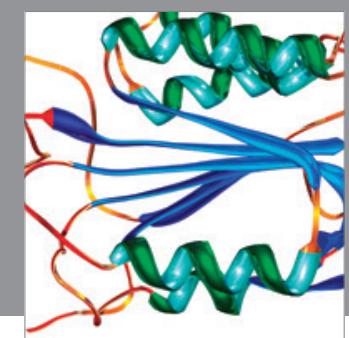

Disease Markers
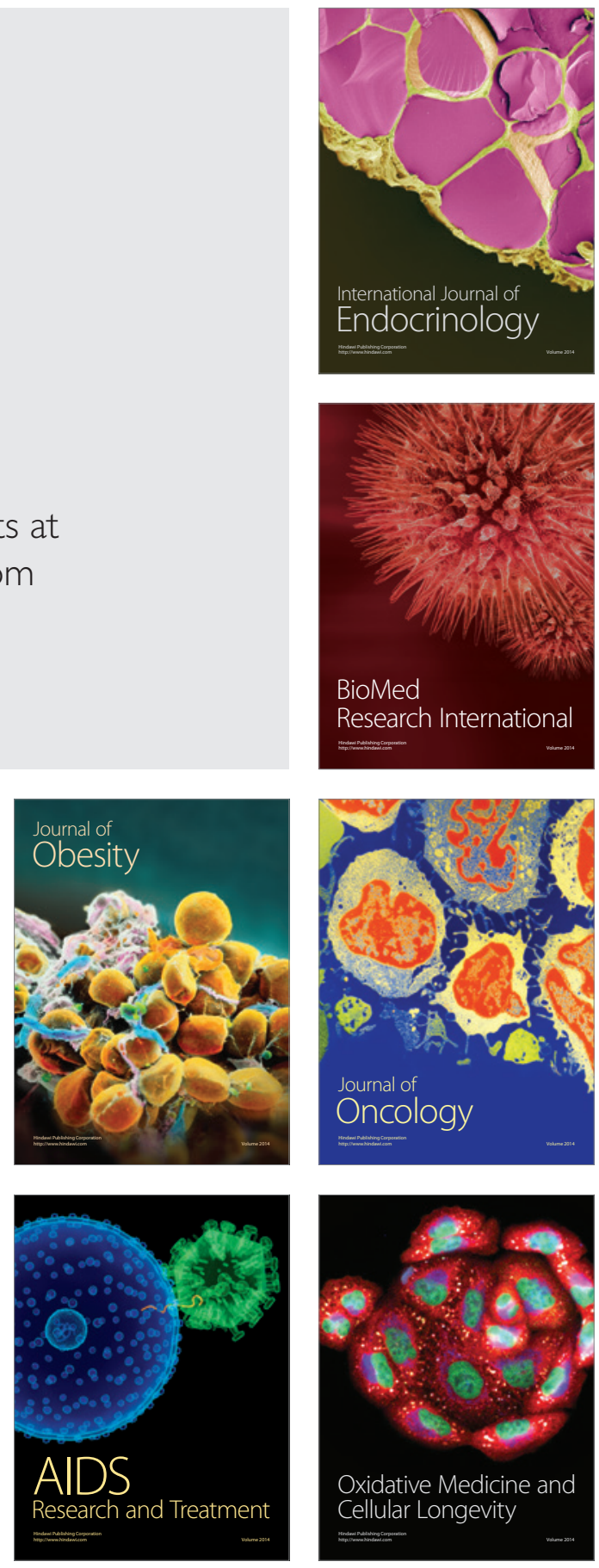